\title{
Methodische Untersuchungen zur Bestimmung der freien Harn-Corticoide mit der Proteinbindungsmethode
}

\author{
Von J. KöbBerling und A. v. z. MÜHLEN \\ Medizinische Universitätsklinik Göttingen, Arbeitsgruppe Endokrinologie
}

(Eingegangen am 12. Juni/14. August 1972)

\begin{abstract}
Eine für die Bestimmung der Plasma-Corticoide von uns beschricbene Proteinbindungsmethode kann mit leichten Modifikationen zur Bestimmung der freien Harn-Corticoide verwandt werden. Als Ursache des auch von anderen Autoren beschriebenen Phänomens der fehlenden Dosisproportionalität zwischen Urinextrakt und Standardlösung erwies sich die nicht ganz vollständige Trennung zwischen gebundenem und freiem Cortisol durch unspezifische Effekte der Urinextrakte. Ein rechnerischer Ausgleich wäre möglich, für den praktischen Gebrauch ist der Fehler jedoch zu vernachlässigen, wenn die Bestimmungsmethode sehr empfindlich eingestellt und nur eine geringe Menge von Urinextrakt ( $0,05 \mathrm{ml}$ Urin) eingesetzt wird.

Bei einem Bereich der Standardkurve zwischen 0 und 10 ng liegt die Präzision der Bestimmung bei 95\% oder darüber. Die untere Nachweisgrenze beträgt 0,05 ng. Die Streuung in der Serie beträgt 5,9\%, die Streuung von Tag zu Tag 7,6\%. Die Wiederfindung unterschiedlicher dem Urin zugesetzter Cortisolmengen ist von $100 \%$ nicht signifikant zu unterscheiden. Der Normalbereich bei Patienten ohne Störungen der Nebennierenrindenfunktion liegt bei 15,3-83,1 $\mu \mathrm{g} / 24 \mathrm{~h}(\overline{\mathrm{x}} 35,9 \mu \mathrm{g} / 24 \mathrm{~h}$, logarithmische Normalversteilung). Die Werte von Patienten mit Nebennierenrindeninsuffizienz oder Cushing-Syndrom sowie nach Gabe von synthetischen Corticoiden oder DepotACTH zeigen eine vollständige Diskriminierung vom Normalbereich. Damit liegt eine vergleichsweise wenig aufwendige aber präzise und sehr empfindliche Methode bei ausreichender Spezifität zur Bestimmung der freien Harn-Corticoide vor.
\end{abstract}

\section{Study of a protein binding method for the determination of the free urinary corticoids}

A protein binding method described by us for the determination of the plasma corticoids can be applied, with minor modifications, to the determination of free urinary corticoids. The lack of proportionality between the measurements on urine extracts and standard solutions, which has been described also by other authors, is apparently caused by incomplete separation of bound and free cortisol, due to nonspecific effects of the urine extract. A correction could be computed, but in practical applications the error may be ignored, if the determination method is very sensitively adjusted, and only a small amount of urinc extract is used $(0.05 \mathrm{ml})$.

In the range of the standard curve between 0 and $10 \mathrm{ng}$, the precision of the determination is $95 \%$ or better. The lower limit of detection is $0.05 \mathrm{ng}$. The precision in the series is $5.9 \%$. The precision from day to day is $7.6 \%$. The recovery of various quantities of cortisol added to the urine cannot be significantly distinguished from $100 \%$. The normal range in patients without adrenal disturbances is $15.3-83.1 \mu \mathrm{g} / 24 \mathrm{~h}(\overline{\mathrm{x}} 35.9 \mu \mathrm{g} / 24 \mathrm{~h}$, logarithmic normaldistribution). The values from patients with adrenal insufficiency, or CusHiNG's syndrome, as well as after administration of corticoids or depot ACTH, lie completely outside the normal range. Thus, this is a comparatively simple, but precise, very sensitive, and sufficiently specific method for the determination of the free urinary corticoids.

Dạ freie Cortisol im Harn, hier wegen nicht ganz vollständiger Spezifität freie Harn-Corticoide genannt, ist zur Untersuchung der Nebennierenrindenfunktion besonders gut geeignet. Seine Ausscheidung ist im Gegensatz zu der der Gesamt-Corticoide (1) nicht vom Metabolismus des Cortisols, also auch nicht vom Körpergewicht, abhängig. Im Gegensatz $z u$ den PlasmaCorticoiden machen sich Veränderungen der Bindungskapazität nicht bemerkbar, da in der Niere nur der allein wirksame „freie“ nicht an Transcortin gebundene Anteil des Plasma-Cortisols filtriert wird.

Die bisherigen Methoden zur Bestimmung der freien Harn-Corticoide waren entweder $z u$ unspezifisch, wie die einfache fluorometrische Bestimmung im ungereinigten Urinextrakt (2), wobei das freie Harn-Cortisol nur zwischen 10 und $80 \%$ der fluorometrisch erfaßten Substanzen ausmacht und die Ergebnisse daher im Mittel um den Faktor 3 zu hoch sind (3), oder sie waren für die Routine zu aufwendig. Dies gilt sowohl für die dünnschicht- oder glasfaserchromatografische Auftrennung mit anschließender Endpunktbestimmung durch die Porter-SIrber-Reaktion (4-6) oder die Fluoro- metrie (7-15) als auch für die Doppelisotopenmethode (16-19) oder die Gaschromatographie (20). Wesentlich einfacher hingegen ist die Bestimmung der freien HarnCorticoide mit Hilfe der kompetitiven Proteinbindung. Hier bestand zunächst die Schwierigkeit, die z. T. auch für die oben genannten Methoden beschriebenen wurde (6), daß eine sichere Abgrenzung erniedrigter Werte vom Normalbereich nicht möglich war $(21-22)$. In jüngster Zeit wurden jedoch Proteinbindungsmethoden beschrieben $(19,23,24)$ mit denen erniedrigte Werte der freien Harn-Corticoide deutlich als solche erkannt werden können.

Für die Bestimmung der Plasma-Corticoide mit der Proteinbindungsmethode wurden von uns einige Verbesserungen und methodische Vereinfachungen vorgeschlagen (25). Eine besondere Herstellungsweise des ${ }^{3} \mathrm{H}$-Cortisol-Bindungsplasmas erlaubt danach eine $\mathrm{Ab}$ lesung in sehr niedrigen Bereichen. Diese Methode, die sich durch eine gute Reproduzierbarkeit und eine geringe Störanfälligkeit bei relativ geringem Arbeitsaufwand auszeichnet, läßt sich mit einigen Veränderungen auch für die Bestimmung der freien Harn-Corticoide 
anwenden. Die Plasmaextraktion und die Reinigung des Extraktes wurden mit geringen Modifikationen von Hsu und Bledsoe (19) übernommen.

Ein bisher ungelöstes Problem war die fehlende Dosisproportionalität von Plasmaextrakt und Standardkurve (19). Größere Extraktmengen lieferten regelmäßig relativ niedrigere Ergebnisse als an derselben Standardkurve abgelesene kleinere Extraktmengen. Die Ursache dieses auch von uns beobachteten Phänomens sollte durch eine Reihe von Untersuchungen geklärt werden. Dabei war das Bestreben, diesen Störfaktor ohne zusätzliche Erhöhung des methodischen Aufwands so gering wie möglich zu halten.

\section{Material und Methoden}

\section{Reagenzien}

Dichlormethan „Uvasol“" (Merck AG, Darmstadt).

Äthanol absolut (Merck AG, Darmstadt).

Aktivkohle reinst (Merck AG, Darmstadt).

Dextran 70 (Pharmacia, Frankfurt).

Insta-Gel (Packard Instrument Comp., Frankfurt).

Hydrocortison (Schering AG, Berlin).

$\left[1,2^{3} \mathrm{H}\right]$ Hydrocortison $20-30 \mathrm{Ci} / \mathrm{mmol}$ (NEN-Chemicals; Dreieichenhain).

Phosphatpuffer $0,25 \mathrm{~mol} / 1, \mathrm{pH} 7,4$.

$\mathrm{NaOH}, 0,1 \mathrm{~mol} / \mathrm{l}$.

Essigsäure, 0,1\%.

Dextran-Holzkohle-Aufschwemmung:

$\begin{array}{ll}\text { Dextran } & 0,05 \\ \text { Aktivkohle } & 0,5\end{array}$

Phosphatpuffer ad $150 \mathrm{ml}$

(bei $+4^{\circ} \mathrm{C}$ mehrere Wochen haltbar).

\section{Cortisol-Standard}

Hydrocortison $1 \mathrm{mg} / 1$ in absolutem Athanol, in kleinen Mengen vor Gebrauch auf $50 \mu \mathrm{g} / 1$ verdünnen.

\section{${ }^{3} H$-Cortisol-Bindungsplasma}

Die Herstellung des ${ }^{3} \mathrm{H}$-Cortisol-Bindungsplasmas erfolgt wie beschrieben (25). Für die Bestimmung der freien Harn-Corticoide empfiehlt sich jedoch anstelle der Plasmaverdünnung von 1:150 eine Verdünnung von 1:250.

\section{Vorgang}

Alle Glassachen müssen gründlich gereinigt und mit Alkohol gespült sein. In Extraktionsgläsern mit Schliffstopfen wird 0,5 oder $1 \mathrm{ml}$ Urin mit Dichlormethan im Verhältnis 1:10 durch $30 \mathrm{~s}$ Schütteln mit dem Vortex-Mischer extrahiert. Die Urinphase wird mit der Wasserstrahlpumpe abgesaugt. Anschließend wird der Extrakt mit je $1 \mathrm{ml} \mathrm{0,1} \mathrm{mol} / 1 \mathrm{NaOH}, 1 \mathrm{ml} 1$ proz. Essigsäure und $1 \mathrm{ml}$ dest. Wasser gewaschen, wobei die wäßr. Phasen jeweils abgesaugt werden. Von dem gewaschenen Extrakt wird ein Anteil von $0,5 \mathrm{ml}$ mit einer Konstriktionspipette in ein Zentrifugenglas überpipettiert. In weitere Zentrifugengläser werden $0,20,50$, 100 und $200 \mu$ der Standardlösung (0, 1, 2,5, 5 und $10 \mathrm{ng}$ Cortisol) pipettiert. Alle Standards und Urinextrakte werden als Doppelbestimmungen angesetzt. Die maximale Anzahl der Einzelproben wird begrenzt durch die Größe der Zentrifugeneinsätze. Die Standardwerte und die Urinextrakte werden im Luftstrom zum Trocknen gebracht. Hierzu wird ein aus Glas gefertigter Verteilerrechen für den Luftstrom, passend für die Zentrifugengläser im Reagenzglasständer, verwandt. Anschließend wird in jedes Glas mit einer Repetierspritze („Ultra-Asept“", $2 \mathrm{ml}, \mathrm{Fa}$. Henke, Tuttlingen) $1 \mathrm{ml}$ der ${ }^{3} \mathrm{H}$-Cortisol-Plasmalösung eingegeben. Alle Gläser werden einige Sekunden mit dem Vortex-Mischer und anschließend $5 \mathrm{~min}$ bei $37^{\circ} \mathrm{C}$ im Wasserbad geschüttelt. Die Proben

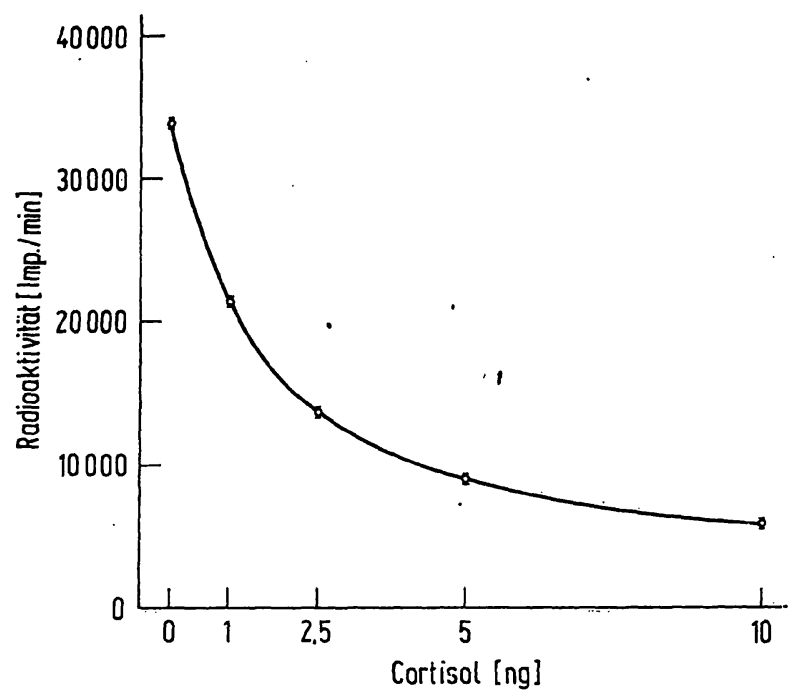

Abb. 1

Cortisol-Standardkurve (12-fach-Bestimmungen) mit Standardabweichungen $(\sigma)$

werden dann für 20 min im Eiswasser inkubiert. Nach dieser Zeit wird mit der Repetierspritze $0,5 \mathrm{ml}$ der Dextran-Holzkohle-Aufschwemmung pipettiert. Dieser Pipettiervorgang muß so schnell wie möglich erfolgen. Die Kohleaufschivemmung mit einem Magnetkern steht während dieser Zeit ebenfalls in einem Eisbad auf einem automatischen Magnetrührer. Auch die Repetierspritze wird im Eisbad vorgekühlt. Vor der ersten Probe wird mindestens $20 \mathrm{mal}$ leer pipettiert. Anschließend werden die Proben im Ständer gemeinsam einige Sekunden kräftig mit der Hand geschüttelt und noch weitere $10 \mathrm{~min}$ im Eisbad gekühlt. Während dieser Zeit werden auch die Zentrifugeneinsätze vorgekühlt (die Vorkühlung kann bei Vorhandensein einer Kühlzentrifuge entfallen). Die Proben müssen dann sofort gleichzeitig etwa $5 \mathrm{~min}$ bei etwa $2000 \mathrm{~g}$ zentrifugiert werden. Auch nach dem Zentrifugieren werden die Zentrifugeneinsätze mit den Proben wieder ins Eisbad gestellt. Von dem Uberstand werden $0,5 \mathrm{ml}$ mit einer EppendorfMikroliterpipette in ein Plastikgefäß mit $10 \mathrm{ml}$ Insta-Gel als Szintillationslösung pipettiert. Gezählt wird nach $30 \mathrm{~min}$ Vorkühlung mit einer Zeitvorgabe von $1 \mathrm{~min}$ oder.einer Impulsvorgabe von 50000 . Eine nach dieser Methode erstellte Standardkurve (12-fach-Bestimmungen) mit den Standardabweichungen $(\sigma)$ ist in Abbildung 1 wiedergegeben. Die an der Standardkurve abgelesenen $\mathrm{ng}$-Werte werden mit 20 multipliziert $(0,5$ von $10 \mathrm{ml}$ Extrakt pro Ansatz) und ergeben dann den Gehalt an freiem Cortisol in ng/ml bzw. $\mu \mathrm{g} / 1$ Urin. Zur Berechnung der 24-StundenAusscheidung wird dieser Wert mit der Urinmenge multipliziert.

\section{Ergebnisse \\ Untersucbungen zur Frage der feblenden Dosis-Proportio- nalität}

Zur Prüfung der Dosis-Proportionalität wurden von einem Extrakt eines Mischurines. (Extraktionsverhältnis $1: 10)$ verschiedene Mengen in die Bestimmung eingesetzt und aus den abgelesenen Werten wiurde eine Kurve gezeichnet. Eine im gleichen Ansatz bestimmte Standardkurve wurde in einer willkürlichen Relation der Maßstäbe ungefähr parallel zu der Kurve aus dém Urinextrakt gezeichnet (Abb. 2). Die Abweichungen zwischen den beiden Kurven wurden über den ganzen Bereich berechnet und in Prozent angegèben. Es zeigt sich, daß die relativen Abweichungen an den verschiedenen Bereichen der Kurven verschieden sind (Abb. 3). Die Kurven verhalten sich so zueinander, daß 


\section{Für Ihr Gerinnungslabor}

\section{Platelin Plus Activator}

Plättchenfaktor-Reagenz mit Celit zur Durchführung der aktivierten PTT

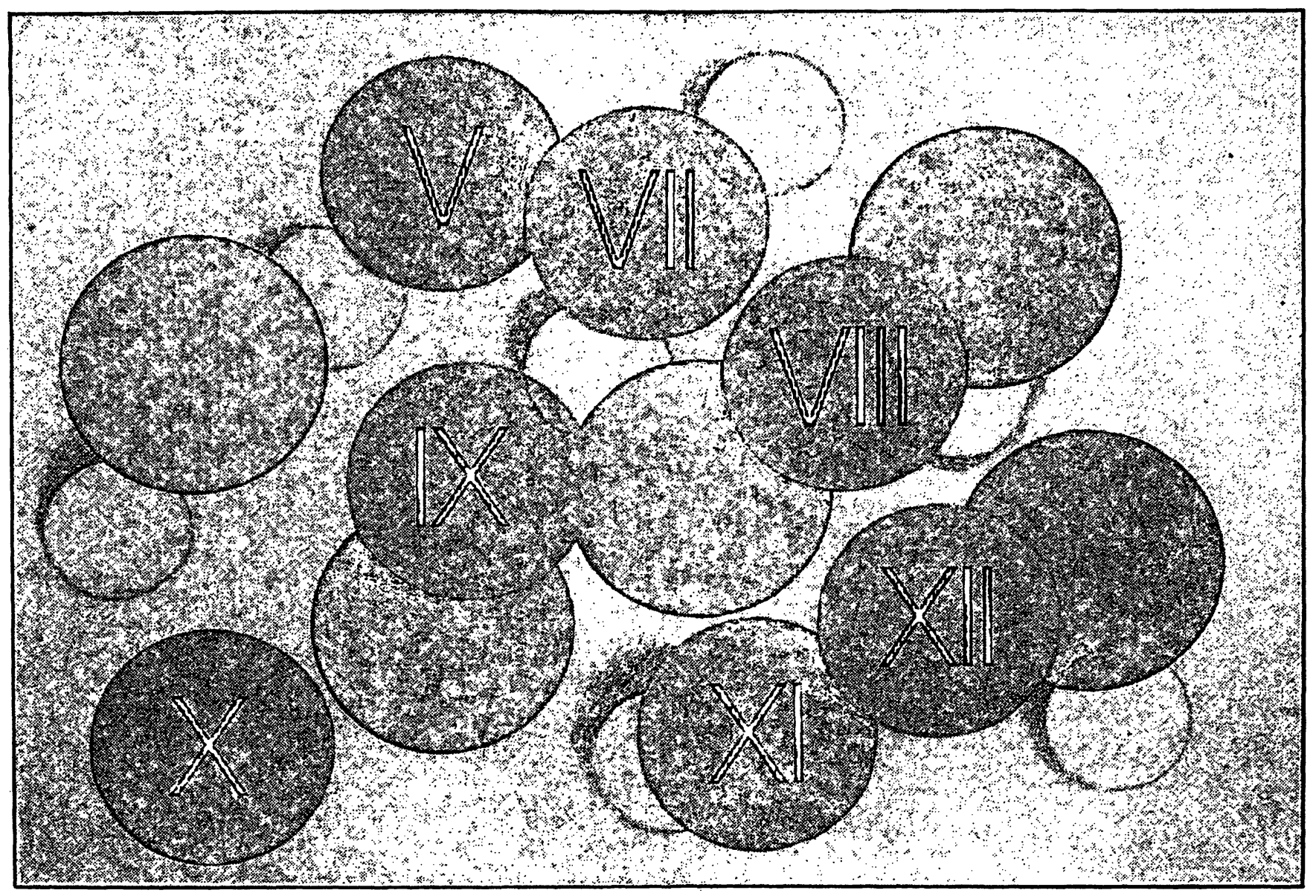

Faktoren-Mangelplasmen

Reagenzien zur quantitativen Bestimmung der Gerinnungsfaktoren (B)(100(ख)(ख)

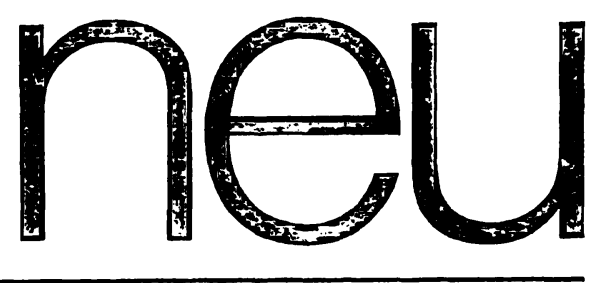




\section{ionenselektiv messen ist einfach und bięet viele neue Möglichkeiten}

Heute schon ist es in vielen Labors wünschenswert, lonenaktivitäten messen zU können. Dafür stehen universelle Geräte zur Verfügung, die den höheren Anforderungen gerecht werden - für alle lieferbaren ionenselektiven Elektroden, z. B. zur Messung von $\mathrm{pCl}-, \mathrm{pCN}$-, $\mathrm{pCa}$ - und $\mathrm{pNH}_{4}-$ Werten. Selbstverständlich lassen sich damit auch pH-Werte und Redoxpotentiale messen. AuBerdem - und das ist interessant - sind diese Geräte zusätzlich für amperometrische Titrationen eingerichtet. Der finanzielle Aufwand ist gering, der Zuwachs an Anwendungsmöglichkeiten und Flexibilität dagegen erfreulich hoch.

Das Ionenaktivitäts-Meßgerät PW 9413 für ionenspezifische Messungen in Industrie und Forschung, z. B. bei der Wasser- und Abwasserüberwachung und -untersuchung - dabei sind kontinuierliche Kontrollmessungen möglich - und
Sogar bei amperometrischen Titrationen genügt ein Knopfdruck. Das Meßgerät zeigt dann den Strom in $\mu \mathrm{A}$ an - direkt geeignet für Karl Fischer Titrationen. Die Gleichspannung ist einsteilbar zwischen 0 und $100 \mathrm{mV}$.

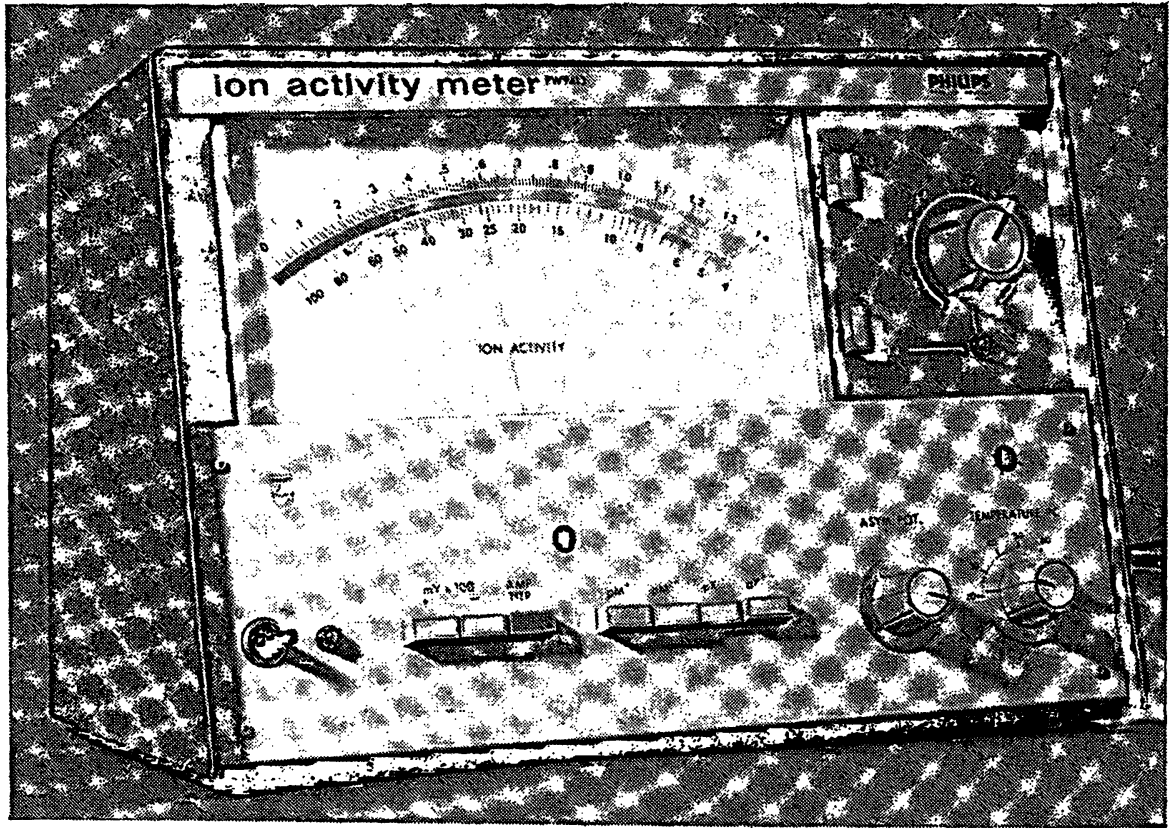

in der klinischen Chemie, z. B. bei der Blut-, Serum-, Speichel- und Harnuntersuchung:

Das Gerät hat eine hohe Empfindlichkeit Dabei ist die Ablesegenauigkeit auf der $190 \mathrm{~mm}$ langen Skala bereits ungewöhnlich gut; sie läßt sich durch Bereichsspreizung noch vergrößern.

... und eine hohe Stabilität

Dafür sorgt der Brückeneingang mit Varaktordioden.

\section{Die Bedienung ist einfach}

Die Bedienungselemente sind übersichtlich angeordnet. Das macht die Arbeit leicht und bequem.

Ein Knopfdruck genügt,

um ein- oder zweiwertige Anionen und Kationen vorzuwählen. Die Ionenaktivität bzw: -konzentration wird dann direkt auf dem Meßgerät abgelesen. normierter Stromausgang

Neben dem Spannungsausgang $0 \ldots$ $140 \mathrm{mV}$ steht ein normierter Stromausgang $0 \ldots 20 \mathrm{~mA}$ zur Verfügung, der vom Eingang galvanisch getrennit ist und zur Ansteuerung von Folgegeräten, wie z. B. Reglern und Schreibern dient.

Meßbereich normal: $0 \ldots 14 \mathrm{p}$-Einheiten, bzw. $0 \ldots \pm 1400 \mathrm{mV}$; gespreizt: 1,4 pEinheiten, bzw. $\pm 140 \mathrm{mV}$, in 14 Stufen um jeweils eine p-Einheit bzw. $100 \mathrm{mV}$ Eingangswiderstand $10^{13} \mathrm{Ohm}$ bei $25^{\circ} \mathrm{C}$ Asymmetriepotential-Einstellung:

$-300 \ldots+300 \mathrm{mV}$

Steilheitskorrektur: $54,0 \ldots 59,2 \mathrm{mV}$ für einwertige und $27,0 \ldots 29,6 \mathrm{mV}$ für zweiwertige lonen

Temperaturkompensation von Hand oder automatisch mit Pt 100 Ohm Widerstandsthermometer

Reproduzierbarkeit $\pm 0,2 \%$ vom Skalenendwert ionenselektive Elektroden

Philips bietet ein gut sortiertes Programm an Elektroden mit hoher Selektivität, hoher Empfindlichkeit und hoher Stabilitäț

mit Festkörpermembran

Chlorid, Bromid, Jodid, Cyanid, Sulfid,

Fluorid, Silber, Cadmium

mit Flüssigmembran

Kalium, Ammonium, Calcium mit Glasmembran

Kalium, Natrium - auch EinstabMeßketten

Referenzelektroden mit Elektrolytbrücke, speziell für Messung mit ionenselektiven Elektroden

Weitere Elektroden, z. B: für Schwermetalle, sind in Vorbereitung

zur weiteren Information schickt Ihnen Philips gern ausführliches

Informationsmaterial. Bitte fordern Sie es an.

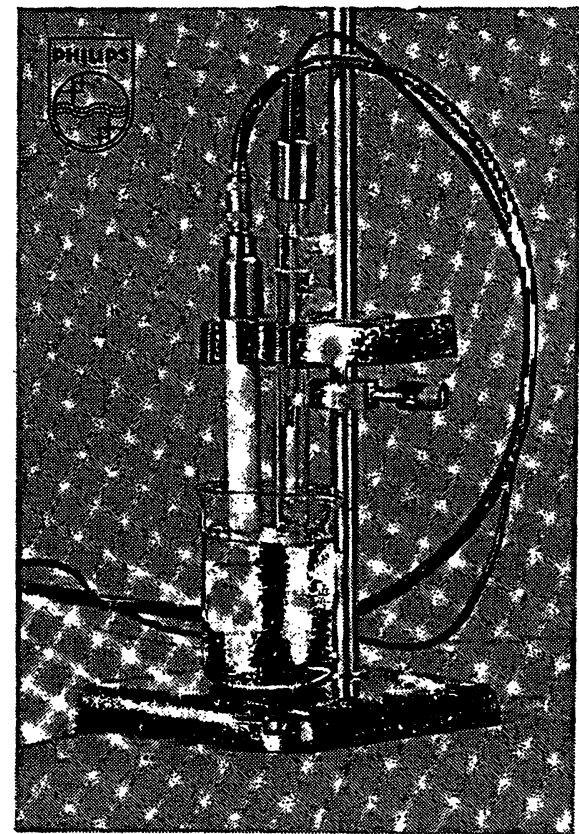

Philips Elektronik Industrie $\mathrm{GmbH}$ 2000 Hamburg 63, Röntgenștraße 22 Telefon (0411) 580131

Telefon-Nummern der Büros in: Berlin (0311) $24590 \mathrm{~g}$, Bielefeld (0521) 23081 81-87, Dortmund (0231) 41961, Düsseld orf (0211) Hannover' (051) 166 01, Kial (0131) 73 Hamburg (0411) 501031 Mannheim (0621) 420, is -18, München (0811) 76791, Nürnberg
(0911) 464763 , Stuttgert (0711) $589081-83$. in Österreich: Österreichische Philips Industrie GmbH, Wien, Triester Str. 64 in der Schweiz: Philips AG, Zürich, Postfach, Tel.: (051) 442211

\section{PHILIPS}

Wir interessieren - uns für das Meßgerät PW 9413 und für ionenselektive Elektroden und bitten um

$\square$ Zusendung ausführlicher Unterlagen

$\square$ ein Angebot:

Gewünschtes bitte ankreuzen oder ergänzen 


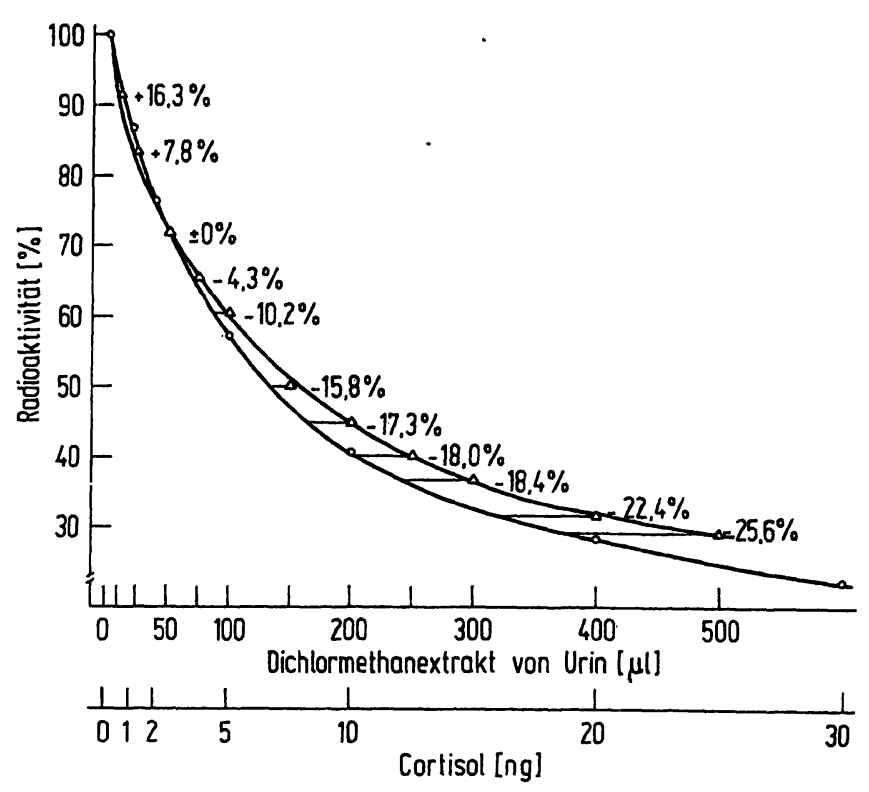

Abb. 2

Dosis-Wirkungskurve eines Urinextraktes $(\Delta-\Delta)$ verglichen mit einer im gleichen Ansatz bestimmten Standardkurve von Cortiso $\left(0_{-0}\right)$. Die Maßstäbe wurden willkürlich so gewählt, daß die Kurven
sich im niedrigen Bereich etwa decken

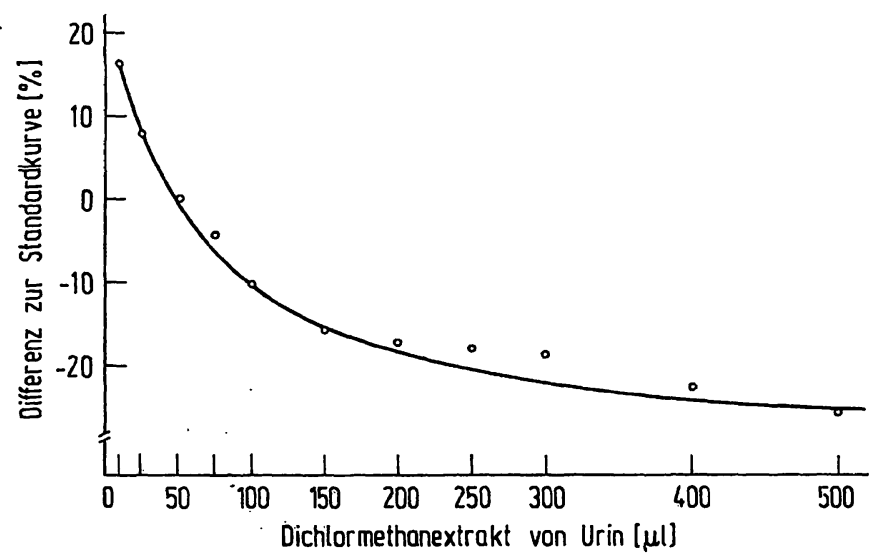

Abb. 3

Die relativen Abweichungen zwischen den beiden Kurven der Abbildung 2 in Abhängigkeit von der Menge des Urinextraktes

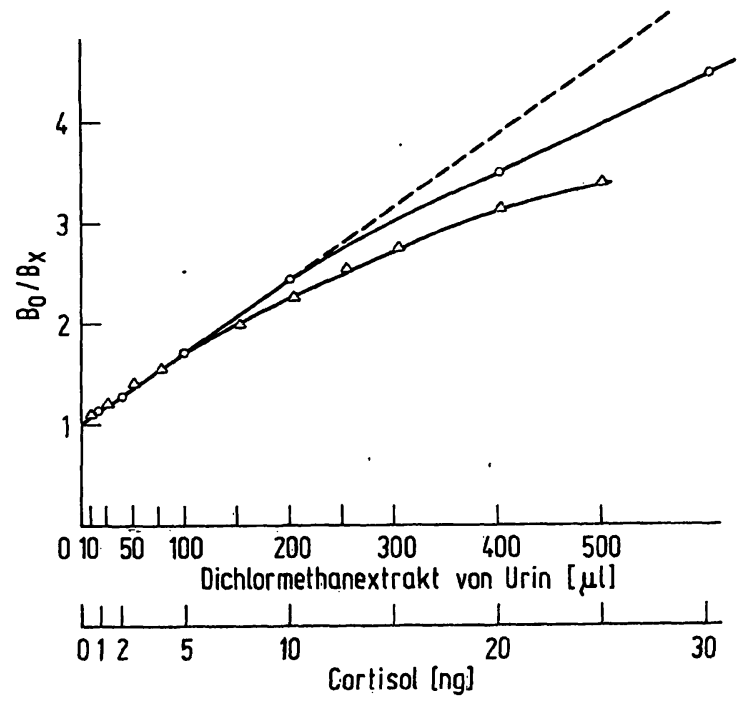

Abb. 4

Die gleichen Kurven wie in Abbildung 2 in der reziproken Darstel-

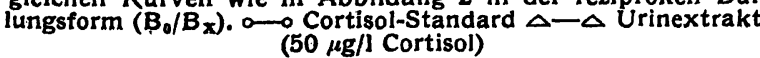

bei hohen Mengen von Urinextrakt ein relativ niedrigerer Cortisolgehalt abgelesen wird. Im Beispiel der Abbildung 2 wurde bei Einsatz von $1 \mathrm{ml}$ Extrakt (entsprechend $0,1 \mathrm{ml}$ Urin) ein gegenüber $0,5 \mathrm{ml}$ Extrakt um $10,2 \%$, bei Einsatz von 2 'ml Extrakt sogar um $17,2 \%$ niedrigerer Wert abgelesen.

Dieselben Zusammenhänge können auch in anderer Form dargestellt werden. Für die Abbildung 4 wurde die Radioaktivität am 0-Punkt der Kurve $\left(B_{0}\right)$ durch die Aktivitäten an allen Einzelpunkten $\left(B_{x}\right)$ geteilt. Die Kurven der Abbildungen 1 und 4 sind also reziprok. Es fällt auf, daß bei dieser Darstellungsweise auch die Standardkurve im hohen Bereich von der theoretisch zu erwartenden Geraden abweicht. Diese Abweichung ist jedoch bei der Kurve des Urinextraktes wesentlich ausgeprägter. Aufgrund theoretischer Überlegungen (s. u.) ist eine Abweichung von der Geraden zu erwarten, wenn sich zu der "Verdrängungskurve“ mit abnehmender Radioaktivität bei zunehmender Menge eingesetzten nicht markierten Cortisols eine andere Kurve mit konstanter Radioaktivität (z. B. ein ZählLeerwert) oder ansteigender Radioaktivität mit zunehmender Menge nicht markierten Cortisols addiert. Der Zähl-Leerwert ist so gering, daß er für den beobachteten Effekt nicht in Frage kommt.

Eine überlagernde Kurve mit ansteigender Tendenz oder eine konstante Radioaktivität wäre zu erwarten, wenn sich im Bindungsplasma ein Protein mit vergleichsweise zum Transcortin geringer Bindungsaffinität und hoher Bindungskapazität befände, welches im üblichen Bereich also noch nicht vollkommen abgesättigt ist. Es wurde geprüft, ob Albumin einen solchen Effekt ausüben könne. Hierzu wurde dem Bindungsprotein Humanalbumin in einer Menge von $30 \mathrm{~g} / 1$ und $120 \mathrm{~g} / \mathrm{l}$ zugesetzt. Damit wurde also die Albuminkonzentration etwa verdoppelt bzw. verfünffacht. Standardkurven mit diesem Bindungsplasma waren nicht von Standardkurven mit Bindungsplasma ohne Albuminzusatz zu unterscheiden.

Eine störende Überlagerung der „Verdrängungskurve“ wäre ebenfalls zu erwarten, wenn der freie Anteil des Cortisols nicht vollständig an die Holzkohle gebunden wird. Zur Prüfung dieser Frage wurden markiertes und unmarkiertes Cortisol ohne Zusatz von Protein im Puffersystem mit Holzkohle versetzt und die Aktivität im Überstand gemessen. Es zeigte sich, daß 1-2,4\% der eingegebenen Aktivität nicht gebunden wurden, also im Puffer in Lösung blieben. Die Bindung an die Holzkohle war geringgradig zeitabhängig. Sie betrug nach 5 min Inkubation 98,2\%, nach 10 min Inkubation $98,75 \%$ und nach 25 min Inkubation $98,87 \%$. Sie ist (im in Frage kommenden Bereich) nicht abhängig von der absoluten Menge des Cortisols oder der spezifischen Aktivität.

Es wurde geprüft, ob der Grad der Holzkohlebindung von dem zugeführten Urinextrakt beeinflußt wird. Dazu wurden bei dem Bindungsversuch ohne Plasmazusatz verschiedene Mengen Urinextrakt zugefügt. Das in dem Urinextrakt enthaltene Cortisol ist dabei ohne 


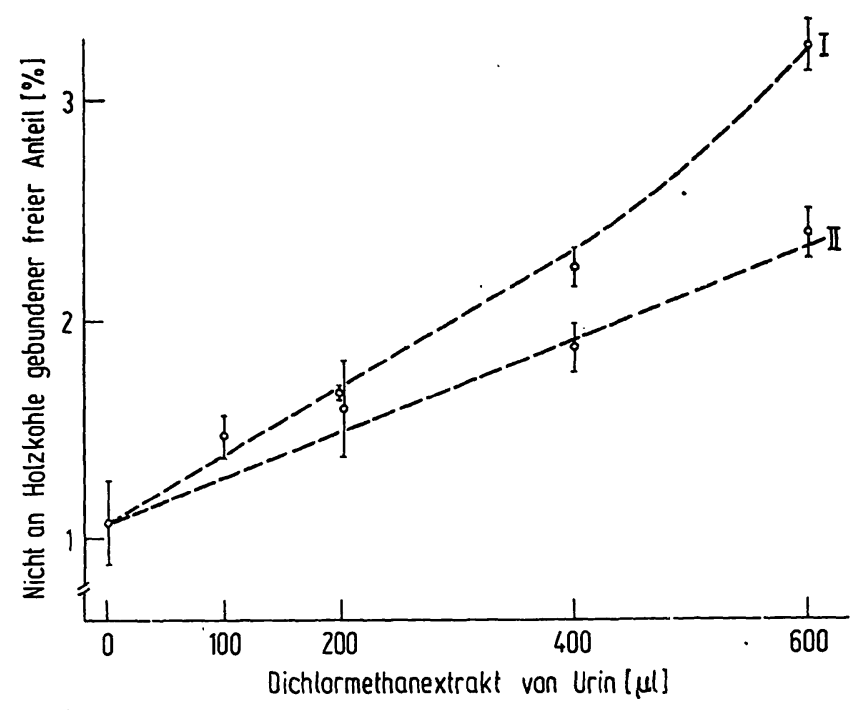

Abb. 5

Einfluß des Urinextraktes auf den Anteil nicht an Holzkohle gebundenen freien Cortisols im Inkubationssystem ohne Bindungsprotein

I Mischurin aus Urinfluß unter $40 \mathrm{ml} / \mathrm{min}$ II Mischurin aus Urinfluß über $40 \mathrm{ml} / \mathrm{min}$

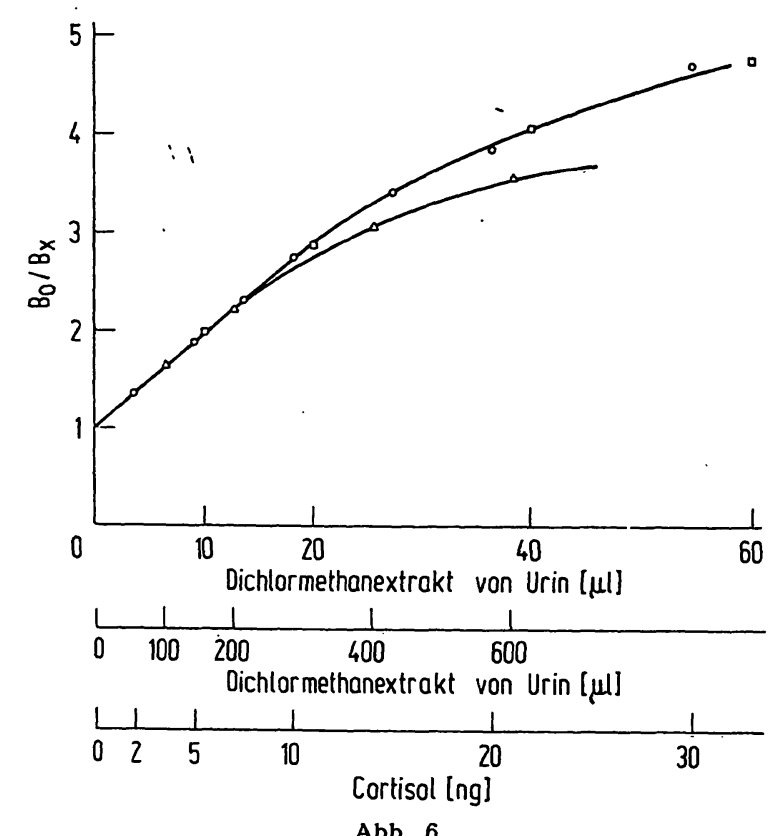

Kurven von je einem Urinextrakt mit niedrigem $(\Delta-\Delta, 35 \mu \mathrm{g} / \mathrm{l})$ und mit hohem $(\square-\square, 550 \mu \mathrm{g} / \mathrm{l})$ Cortisolgehalt im Vergleich zu einer Standardkurve ( $(-0)$. Die Abweichung von der Standardkurve ist abhängig von der Menge des zugefügten Urinextraktes, gewählt, daß die Kurven sich im niedrigen Bereich etwa decken

Einfluß. Die Abbildung 5 zeigt, daß mit zunehmender Menge des Urinextraktes die Bindung des Cortisols an die Holzkohle abnimmt. Ein k konzentrierterer (,hochgestellter") Urin hat einen stärkeren Einfluß als ein verdünnterer („,niedriggestellter ${ }^{\text {“) }}$ Urin. Der Effekt, der zu der Abweichung von der Geraden führt, nimmt also mit zunehmender Menge des eingesetzten Urins $z u$. Damit wird verständlich, daß die Kurve des Urinextraktes sich zunehmend weiter von der Standardkurve entfernt.

Die Hemmung der Cortisolbindung an die Holzkohle durch einen im Urinextrakt vorhandenen Störfaktor ist also die Ursache für die fehlende Dosisproportionalität mit der Standardkurve. Versuche weiterer Reinigungs- schritte des Urinextraktes durch Waschen führten nicht $\mathrm{zu}$ einer Verringerung dieses Störfaktors. Demnach ist zu erwarten, daß die Abweichung von der Standardkurve bei höheren Cortisolmengen im Urin, bei also vergleichsweise geringeren Extraktmengen, geringer wird. Dies kann in der Abbildung 6 demonstriert werden. Die Maßstäbe der beiden Urinextrakte und der Standardkurven wurden so gewählt, daß die Kurven im niedrigen Bereich zusammenfallen, Ein Mischurin mit $35 \mu \mathrm{g}$ Cortisol/l zeigt die typische Abweichung von der Standardkurve im hohen Bereich. Ein Urin von einer Patientin mit Nebennierenrindenkarzinom mit einem Cortisolgehalt von $550 \mu \mathrm{g} / \mathrm{l}$ weicht in dem getesteten Bereich nicht von der Standardkurve ab.

\section{Untersucbungen zur Qualität der Metbode}

Aus den 12-fach-Bestimmungen der Standardkurve (Abb. 1) läßt sich die Präzision für die einzelnen Bereiche angeben. Die Variationskoeffizienten in Prozent berechnen sich nach VK $=s_{\mathbf{x}} / \overline{\mathbf{x}} \cdot 100$, die Präzision beträgt $100-\mathrm{VK}$. In der Tabelle 1 wurden die Variationskoeffizienten für Kurven mit der Plasmaverdünnung 1:250 wiedergegeben. Wie aus der Tabelle hervorgeht, liegt die Präzision in allen Bereichen etwa bei $95 \%$ oder darüber.

Zur Berechnung der unteren Nachweisgrenze wurde die 2-fache Streuung $\sigma$ (Standardabweichung) der Werte am 0-Punkt der Kurve zugrunde gelegt. Der an dem Punkt $\mathrm{B}_{0}-2 \sigma$ der Kurve abgelesene Wert entspricht der geringsten Menge, die unter Zugrundelegung von $2 \sigma$-Grenzen signifikant von $0 \mathrm{zu}$ unterscheiden ist. Sie beträgt in dem angegèbenen Versuch $0,050 \mathrm{ng}$. Da bei der beschriebenen Art der Extraktion mit einfacher Reinigung des Extraktes ohne Chromatographie keine Urin-Leerwerte auftreten, beträgt die untere Nachweisgrenze im Urin bei Einsatz der üblichen Extraktmenge $1 \mu \mathrm{g} / \mathrm{l}$.

Zur Bestimmung der Präzision der Gesamtbestimmung innerhalb eines Versuches („Streuung in der Serie") wurde ein Mischurin mit einem mittleren Cortisolgehalt von $59,4 \mu \mathrm{g} / 110 \mathrm{mal}$ extrahiert. Von jedem Extrakt wurde je drei mal 0,5 $\mathrm{ml}$ in die Endpunktbestimmung eingesetzt. Die Streuung aller Einzelwerte betrug $\pm 3,33 \mu \mathrm{g} / \mathrm{l}$, der Variationskoeffizient also 5,6\%. Innerhalb der Dreifachbestimmungen betrug der Variationskoeffizient $4,8 \%$. Werden die Varianzen dieser beiden Streuungsmaße subtrahiert, ergibt sich für die Extraktion ein Variationskoeffizient von $\sqrt{5,6^{2}-4,8^{2}}=$

Tab. 1

Präzision der Standardkurve bei 12-fach-Bestimmungen in verschiedenen Bereichen

\begin{tabular}{ccc}
$\begin{array}{c}\text { Cortisol } \\
\text { (ng) }\end{array}$ & o(ng) & VK (\%) \\
\hline 0 & $\pm 0,025$ & - \\
1 & $\pm 0,054$ & 5,4 \\
2,5 & $\pm 0,073$ & 3,0 \\
5 & $\pm 0,155$ & 3,1 \\
10 & $\pm 0,202$ & 2,0 \\
\hline
\end{tabular}


Tab. 2

Wiederfindung von zugesetztem Cortisol aus einem Mischurin (10-fach-Bestimmungen)

\begin{tabular}{cccc}
\hline Zusatz & $\begin{array}{c}\text { Cortisol } \\
(\mu \mathrm{g} / \mathrm{l})\end{array}$ & $\sigma(\mu \mathrm{g} / \mathrm{l})$ & Wiederfindung \\
\hline ohne & 44,9 & $\pm 1,37$ & - \\
$20 \mu \mathrm{g} / \mathrm{l}$ & 65,1 & $\pm 2,08$ & $20,2: 20=101 \%$ \\
$50 \mu \mathrm{g} / \mathrm{l}$ & 96,1 & $\pm 2,29$ & $51,2: 50=102,4 \%$ \\
$100 \mu \mathrm{g} / \mathrm{l}$ & 144,9 & $\pm 2,56$ & $100,0: 100=100,0 \%$ \\
\hline
\end{tabular}

2,9\%. Bei einem zweiten Mischurin mit 35,6 $\mu \mathrm{g} / \mathrm{l}$ wurde bei 12-fach-Bestimmungen eine Streuung von $1,9 \mu \mathrm{g} / 1$ gefunden, also ein Variationskoeffizient von 5,9\%.

Zur Bestimmung der Präzision der Methode bei verschiedenen Versuchen an verschiedenen Tagen („Streuung von Tag zu Tag') wurde der Mischurin mit einem Cortisolgehalt von $35,6 \mu \mathrm{g} / \mathrm{l}$ bei $-18^{\circ} \mathrm{C}$ eingefroren und bei 17 verschiedenen Versuchen eingesetzt. Die Streuung betrug $2,6 \mu \mathrm{g} / \mathrm{l}$, der Variationskoeffizient demnach $7,3 \%$.

Als einzelner Teilschritt wurde die Präzision des $\mathrm{Pi}$ pettierens mit der Repetierspritze geprüft. Die Streuung

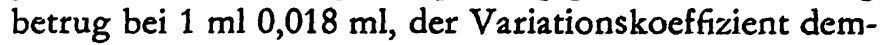
nach $1,8 \%$.

Zur Bestimmung der Wiederfindung wurde ein Mischurin mit einem Cortisolgehalt von $44,9 \mu \mathrm{g} / \mathrm{l}$ je $10 \mathrm{mal}$ extrahiert

1. ohne Zusatz,

2. mit Zusatz von $20 \mu \mathrm{g}$ Cortisol/1,

3. mit Zusatz von $50 \mu \mathrm{g}$ Cortisol/1,

4. mit Zusatz von $100 \mu \mathrm{g}$ Cortisol/1.

Von den Extrakten wurde in üblicher Weise $0,5 \mathrm{ml}$ in die Endpunktbestimmung eingesetzt. Die Tabelle 2 zeigt die dabei gemessenen Cortisolwerte und die jeweiligen Wiederfindungsraten. Die Wiederfindung beträgt also im Mittel 101,1\%. Hieraus ergibt sich auch, $\mathrm{da}$ die Dosisproportionalität bei Ablesung von verschiedenen Punkten der Standardkurve nicht eingeschränkt ist, wenn sich nicht gleichzeitig die Extraktmenge ändert.

Zur Frage der Haltbarkeit der freien Corticoide im Harn wurde ein Mischurin 12 Tage bei Raumtemperatur stehengelassen. In zweitägigen Abständen wurden Proben dieses Mischurines eingefroren, die Bestimmungen erfolgten anschließend in einem Versuch. Über 6 Tage war der Gehalt an freien Corticoiden konstant, vom 8. Tag an kam es zu einer Zunahme, die am 10. Tag deutlich ausgeprägt war ( $148 \%$ des Ausgangswertes). $\mathrm{Zu}$ dieser Zeit war der Urin nach Aspekt und Geruch bereits deutlich bakteriell zersetzt.

\section{Freie Harn-Corticoide in 24-Stunden-Urinen}

181 24-Stunden-Urine von 121 Probanden ohne Störungen der Nebennierenrindenfunktion wurden untersucht. Es erwies sich, daß nicht die numerischen Werte, sondern deren Logarithmen normal verteilt sind (26). Der Mittelwert beträgt $35,9 \mu \mathrm{g} / 24 \mathrm{~h}$, der $2 \sigma$-Streubereich 15,3 . bis $83,1 \mu \mathrm{g} / 24 \mathrm{~h}$. Zwischen männlichen
(36,6 $\mu \mathrm{g} / 24 \mathrm{~h})$ und weiblichen $(34,6 \mu \mathrm{g} / 24 \mathrm{~h})$ Probanden war kein signifikanter Unterschied festzustellen. Auch Ubergewichtige $(34,7 \mu \mathrm{g} / 24 \mathrm{~h})$ oder Untergewichtige $(42,6 \mu \mathrm{g} / 24 \mathrm{~h})$ unterschieden sich nicht signifikant von Normalgewichtigen $(36,1 \mu \mathrm{g} / 24 \mathrm{~h})$. Dagegen waren die Werte bei 87 ambulanten Personen mit $40,2 \mu \mathrm{g} / 24 \mathrm{~h}$ (17,1-94,2) gegenüber 94 stationären Patienten mit $32,4 \mu \mathrm{g} / 24 \mathrm{~h}(14,3-73,1)$ signifikant erhöht. Alle Einzelwerte nach $G$ abe von synthetischen Corticoiden ( $\mathrm{n}=9$; $1,0-11,6 \mu \mathrm{g} / 24 \mathrm{~h}$ ) oder bei Nebennierenrindenunterfunktion ( $n=20 ; 0,4-13,0 \mu \mathrm{g} / 24 \mathrm{~h}$ ) lagen unter dem unteren Grenzwert. Alle Werte nach Gabe von DepotACTH ( $n=6 ; 255-1500 \mu \mathrm{g} / 24 \mathrm{~h})$ oder bei CushingSyndrom ( $n=29 ; 166-3260 \mu \mathrm{g} / 24 \mathrm{~h}$ ) lagen über dem oberen Grenzwert. Damit findet sich eine vollständige Diskriminierung zwischen Normalbereich und pathologischen Zuständen.

\section{Diskussion}

Die reziproke Darstellung der Kurve (Abb. 4) gründet sich auf die Annahme, daß der Anteil des an Transcortin gebundenen Cortisols sich reziprok proportional zu seiner Konzentration verhält. Es läßt sich daher folgende Gleichung aufstellen:

$$
\frac{\mathrm{B}_{0}}{\mathrm{~B}_{\mathrm{x}}}=\frac{\mathrm{C}_{\mathrm{x}}}{\mathrm{C}_{\mathrm{o}}}
$$

Hicrbei bedeuten:

$\mathrm{B}_{0}$ : Der an Trancortin gebundene Anteil der eingegebenen Radioaktivität (als Anteil der gemessenen Aktivität an der Gesamtaktivität im Ansatz) am 0-Punkt der Kurve, also ohne Zusatz unmarkierten Cortisols.

$B_{\mathbf{x}}$ : Der an Transcortin gebundene Anteil der eingegebenen Radioaktivität (als Anteil der gemessenen Aktivität an der Gesamtaktivität im Ansatz) am Punkt $x$ der Kurve. $\mathrm{Da}$ in dem Quotient $\mathrm{B}_{0} / \mathrm{B}_{\mathbf{x}}$ sich die Gesamtaktivität im Ansat\% wegkürzen läßt, können hier einfach die gemessenen Impulse/min eingesetzt werden.

$\mathrm{C}_{0}$ : Dic Cortisolmenge am Punkt 0 der Kurve, also die im Ansatz enthaltene Menge markierten und unmarkierten Cortisols.

$\mathrm{C}_{\mathbf{x}}$ : Die Cortisolmenge am Punkt $\mathrm{x}$ der Kurve, die sich zusammensetzt aus $C_{0}+x$, wenn $x$ die zugegebene Menge unmarkierten Cortisols bedeutet, also die Standardmenge oder die zu bestimmende Cortisolmenge im Extrakt.

Damit läßt sich die Gleichung umformen in

$$
\frac{B_{0}}{B_{x}}=\frac{C_{0}+x}{C_{0}}=\frac{x}{C_{0}}+1 .
$$

Werden also die Impulse am 0-Punkt der Kurve durch die Impulse an den einzelnen Kurvenpunkten geteilt, ergibt sich eine Gerade, die bei $x=0$ die $y$-Achse bei 1 schneidet. Der Anstieg der Geraden ist ausschließlich von $\mathrm{C}_{0}$ abhängig, also von der Gesamtmenge des im Ansatz enthaltenen markierten und unmarkierten Cortisols. 
Die einfachen Verhältnisse gelten nur unter drei Voraussetzungen:

1. Die Bindung an das Transcortin ist unabhängig von der Menge des zugegebenen Cortisols. Dies ist nur möglich, wenn die maximale Bindungskapazität in jedem Fall überschritten ist.

2. Das markierte und das unmarkierte Cortisol verhalten sich in den Bindungseigenschaften gleich.

3. Es erfolgt eine vollständige Abtrennung des gebundenen vom ungebundenen Cortisol.

Die ersten beiden Voraussetzungen sind für das Cortisol und für das in beschriebener Weise (25) vorbereitete Bindungsplasma wahrscheinlich weitgehend erfüllt. Es besteht zumindest kein Anhalt dafür, daß sich die hier genannten Bedingungen bei der Standardkurve und dem extrahierten Cortisol unterschiedlich verhalten.

Die dritte Voraussetzung ist nach den vorliegenden Untersuchungen nicht erfüllt, denn ein Teil des ungebundenen Cortisols wird nicht an Holzkohle gebunden und damit mit der Fraktion des an Transcortin gebundenen Cortisols mitgemessen. Dieser Anteil ist bei der Standardkurve konstant, bei der Messung des Urinextraktes von der Menge des Extraktes abhängig, also eine Funktion der zu messenden Cortisolmenge. Bei den folgenden Berechnungen wird diese Funktion als linear angenommen, was nach der Abbildung 5 in einem weiten Bereich berechtigt erscheint. Statt des gebundenen Anteils $B_{x}$ wird also ein $B_{x}^{\prime}$ gemessen, das sich um den kleinen Anteil des freien Cortisols erhöht. Es besteht die Beziehung:

$$
B_{x}^{\prime}=B_{x}+(a x+b)\left(1-B_{x}\right)
$$

$\mathrm{Da}_{\mathrm{x}}$ den an Transcortin gebundenen Anteil des Cortisols darstellt, ist $1-B_{\mathbf{X}}$ der nicht an Transcortin gebundene (freie) Anteil. Von diesem freien Cortisol wird der Anteil an ax $+b$ nicht an Holzkohle gebunden. $b$ bedeutet dabei den konstanten Anteil, ax den Anteil, der mit Zunahme der Extraktmenge zunimmt, der also von der $z u$ messenden Cortisolmenge $x$ abhängt.

Da nach Gl. (2) die Beziehung

$$
\mathrm{B}_{\mathrm{x}}=\frac{\mathrm{B}_{0}}{\frac{\mathrm{x}}{\mathrm{C}_{0}}+1}
$$

besteht, kann die Gl. (3) folgendermaßen umgeschrieben werden:

$$
\begin{aligned}
& \frac{1}{B_{x}^{\prime}}=\frac{1}{\frac{B_{0}}{\frac{x}{C_{0}}+1}+(a x+b)\left(1-\frac{B_{0}}{\frac{x}{C_{0}}+1}\right)} \\
& =\left(\frac{x}{C_{0}}+1\right) \frac{1}{B_{0}+(a x+b)\left(\frac{x}{C_{0}}+1-B_{0}\right)}
\end{aligned}
$$

Durch Einsetzen von $\mathrm{x}=0$ wird $\mathrm{B}_{0}^{\prime}$ berechnet, also die wirklich gemessene Aktivität am 0-Punkt der Kurve. Durch Multiplikation mit den durch die Gl. (5) berechneten Werten kann der Quotient $\mathbf{B}_{0}^{\prime} / \mathrm{B}_{\mathbf{x}}^{\prime}$ berechnet werden.

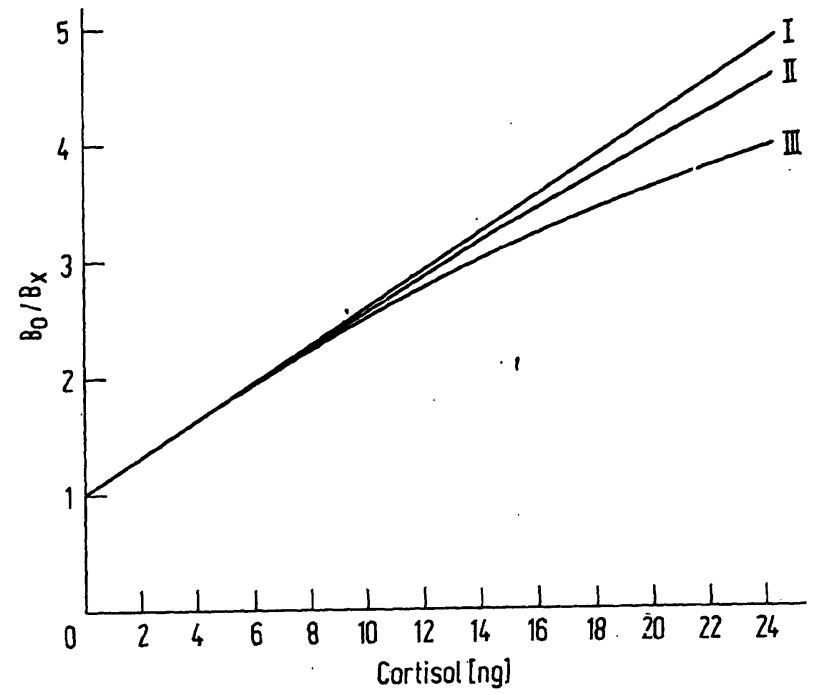

Abb. 7

Nach der Gl. (5) errechnete Kurven für Cortisol-Standard (II) und Urinextrakt (III) sowie die bei vollständiger Trennung von gebundenem und freiem Anteil theoretisch zu erwartende Gerade (I)

Diese Berechnung wurde mit einem Compuiterprogramm für ein Beispiel mit Zahlenwerten durchgeführt, die den in den Versuchen ermittelten etwa entsprechen (Abb. 7). Für diese Kurven wurden folgende Werte eingesetzt: $\mathrm{B}_{0}=0,6, \mathrm{C}_{0}=5,9, \mathrm{~b}$ in Kurve $\mathrm{I}=\mathrm{O}_{2}$ in den Kurven II und III $=0,018$ (d. h. die Bindung an die Holzkohle beträgt nur $98,2 \%$ ), a in den Kurven I und $I I=0$, in der Kurve III =0,0009 (d. h. der Anteil von 1,8\% des nicht an Holzkohle gebundenen Cortisols nimmt pro ng zugefügten Cortisols um $0,09 \% \mathrm{zu}$, würde sich also bei 20 ng verdoppelt haben. Bei einem mittleren Cortisolgehalt von $40 \mu \mathrm{g} / 1$ entspricht dies also einem Extrakt von $0,5 \mathrm{ml}$ Urin).

Die Kurve I entspricht damit einer idealen Darstellung unter der Annahme einer vollständigen Abtrennung von freiem und gebundenem Cortisol. Die Kurve II stellt eine Standardkurve mit konstantem freien nicht an Holzkohle gebundenem Cortisol dar. Die Kurvie III wurde unter der Annahme eines zunehmenden Anteils freien nicht an Holzkohle gebundenen Cortisols errechnet, entspricht also einer Kurve aus Urinextrakt.

Es fällt eine große Ähnlichkeit der Kurven der Âbbildung 4 mit diesen rechnerisch ermittelten Kurven auf. Es erscheint deshalb berechtigt, anzunehmen, daß die unvollständige Bindung an die Holzkohle die wesentliche Ursache für die fehlende Dosis-Proportionalität zwischen Standardkurve und Urinextrakt darstellt. Die fehlende Dosis-Proportionalität ist nicht nur bei Verwendung von Holzkohle zu beobachten, sie wurde auch bei Verwendung von Florisil als Trennmethode beschrieben (19).

Worauf der störende Einfluß des Urins beruht und welcher mit Dichlormethan extrahierbare Faktor des Urins hierfür verantwortlich ist, bleibt unklar. Versuche, ihn chromatographisch abzutrennen, wurden nicht unternommen, weil ein solcher Schritt die Methode in nicht angemessener Form belasten würde. Der unbekannte Urinfaktor könnte entweder zu einer dosisabhängigen Cortisolbindung führen oder et könnte auf 
irgendeine Weise die Bindung des Cortisols an die Holzkohle hemmen.

Theoretisch besteht die Möglichkeit eines rechnerischen Ausgleichs der beschriebenen Störung, indem in jedem Versuch die Faktoren $B_{0}^{\prime}$, a und $b$ mitbestimmt werden. $B^{\prime}$, und a sind nur von der Inkubationszeit der Holzkohle oder von Temperaturschwankungen abhängig, innerhalb eines Versuches also konstant. Der Faktor b müßte aber für jede Urinprobe getrennt bestimmt werden, $\mathrm{da} b$ von der Konzentration des Urins abhängt (Abb. 5). Dies würde die Brauchbarkeit de: Methode erheblich einschränken.

Aus den Ausführungen ergibt sich, daß bei unterschiedlicher Ablesung an verschiedenen Bereichen der Standardkurve immer der Wert mit der geringsten Extraktmenge dem „richtigen“ Wert am nächsten kommt. Für den praktischen Bedarf läßt sich deshalb das Problem umgehen, indem die Methode so empfindlich eingestellt wird, daß die Menge des einzusetzenden Urinextraktes sehr klein wird. Gegenüber der ursprünglichen Beschreibung unserer Methode (25) wurde die Empfindlichkeit durch stärkere Verdünnung des Bindungsplasmas (1:250 statt 1:150) ohne Verlust an Präzision (Tab. 1) erhöht. Bei dieser Empfindlichkeit wird nur der Extrakt von 0,05 ml Urin eingesetzt. Durch diese geringe Extraktmenge erhöht sich der Anteil des nicht an Holzkohle gebundenen freien Cortisols sehr wenig (Abb. 5), macht sich also bei der Gesamtvarianz der Bestimmung praktisch nicht bemerkbar. Lediglich bei sehr geringen Konzentrationen von Cortisol muß u. U. eine größere Menge von Extrakt eingesetzt werden. In diesen Fällen wird aber so weit am Beginn der Standardkurve abgelesen, daß der Störfaktor aus dem Urin sich ebenfalls kaum bemerkbar macht. Bis weit in den Bereich hypoadrenaler Zustände (bis etwa $1 \mu \mathrm{g} / 1$ ) läßt sich noch hinreichend genau ablesen. Die Empfehlung von Hsu und Bledsoe (19), jeden Urinextrakt in drei verschiedenen Mengen einzu- setzen und davon den Mittelwert zu bilden, ist ungünstig, die Mittelwerte liegen dann in der Regel zu niedrig.

Die angegebenen Untersuchungen zur Qualität der Bestimmungsmethode lassen erkennen, da $\beta$ trotz des oben angeführten nur mit Einschränkung gelösten Problems der nicht exakten Dosis-Proportionalität die Methode auch hohen Qualitätsansprüchen genügt. Vergleiche mit anderen Bestimmungsmethoden der freien Harn-Corticoide wurden nicht durchgeführt. Hsu und Br.edsoz (19) fanden sehr gute Übereinstimmungen sowohl mit der Doppelisotopentechnik als auch mit der Phenylhydrazin-Reaktion nach dünnschichtchromatographischer Trennung. $\mathrm{Da}$ unsere Normalbereiche sich von denen der o. g. Autoren nicht unterscheiden, kann von der „Richtigkeit“ der Methode ausgegangen werden.

Auch zur Spezifität liegen keine eigenen Untersuchungen vor. Hier gelten dieselben Einschränkungen wie bei der Bestimmung der Plasma-Corticoide (25).

Der eingeführte Begriff der freien Harn-Corticoide wird beibehalten, obgleich mit der beschriebenen Methode mit Ausnahme weniger pathologischer Zustände fast ausschließlich Cortisol gemessen wird. Cortison, Corticosteron, 11-Desoxycortisol, Progesteron und 17Hydroxyprogesteron werden zwar ebenfalls erfaßt, kommen aber kaum in freier Form im Harn vor. Nur Corticosteron wird in nennenswerter Menge im Harn ausgeschieden, die jedoch auch noch weniger als $10 \%$ des Cortisols beträgt. Mit Dichlormethan wird Corticosteron nur zu etwa $70 \%$ extrahiert (24) und die Bindungsaffinität zum Transcortin liegt im Vergleich zu Cortisol bei etwa 50\%. Damit liegt auch dieser Störfaktor maximal bei wenigen Prozent. Da die beiden genannten Steroide sich bezüglich der Nebennierenrindenfunktion ähnlich verhalten (mit Ausnahme sehr seltener Enzymdefekte) bestehen bezüglich der Spezifität keine zu berücksichtigenden Einschränkungen.

\section{Literatur}

1. Porter, R. H. \& Silber, C. C. (1954), J. Biol. Chem. 210, 923-932. - 2. Matringly, D., Dennis, P. N., Pearson, J. \& Cope, C. L. (1964), Lancet II, 1046-1049. - 3. EsPINER, E. A. (1965), J. Endocrinol. 33, 233--239. - 4. CRABBE, J., REDDY, W. J., Ross, E. J. \& ThorN, G. W. (1958), J. Clin. Endocrinol. 18, 1147-1177. - 5. Ross, E. J. (1960), J. Clin. Endocrinol. 20, 1360-1365. - 6. Rosner, J. M., Cos, J. J., Biglieri, E. G., Hane, S. \& Forsham, P. H. (1963), J. Clin. Endocrinol. 23, 820-827. - 7. Brooks, R. V., Dutre, J., Gogate, A. N., Mills, I. H. \& Prunty, F. T. G. (1963), J. Clin. Endocrinol. 23, 725-736. 8. Gogate, A. N. \& Prunty, F. T. G. (1963), J. Clin. Endoctinol. 23, 747-751. - 9. Gerdes, H. \& StaIB, W. (1965), Klin. Wochenschr. 43, 744-747. - 10. HubL, W. \& BüCHNER, M. (1968), Clin. Chim. Acta 21, 461-467. - 11. Schachinger, V. \& Zicha, L. (1968), Endokrinologie 53, 153-162. - 12. MarTin, M. M. \& Martin, A. L. A. (1969), Clin. Chim. Acta 27, 37.9-383. 13. Stolecke, H. (1970), Horm. Metab. Res. 2, 298-301. 14. Stoliecke, H. (1970), Symp. Deut. Ges. Endokrinol. 16,
379-381. - 15. Usur, T. \& Kawamoto, H. (1970), Clin. Chim. Acta 30, 595-601. - 16. Schedl, H. P., Chen, P. S., Greene, G. \& REDD, D. (1959), J. Clin. Endocrinol. 19, 1223-1229. 17. Beisel, W. R., Cos, J. J., Horton, R., Cheo, P. Y. \& ForshAM, P. H. (1964), J. Clin. Endoctinol. 24, 887-893. - 18. Vagnucci, A. I., Hesser, M. E., Kozak, G. P., Pauk, G. L., Lauler, D. P. \& Thorn, G. W. (1965), J. Clin. Endocrinol. 25, 1331-1339. - 19. Hsu, T. H. \& Bledsoe, T. (1970), J. Clin. Endocrinol. 30, 443-448. - 20. Gerdes, H. \& Teller, W. (1968), Acta Endoctinol. 59, 203-217. - 21. MURPhy, B. E. P. (1968), J. Clin. Endocrinol. 28, 343-348. - 22. BeArdwell, C. G., Burke, C. W. \& Cope, C. L. (1968), J. Endocrinol. 42, 79-89. - 23. Merkle, A. W., Takiguchi, H., Mizutani, S., TyLer, F. H. \&. West, C. D. (1969), J. Lab. Clin. Med. 74, 803 bis 812. - 24. Kolanowski, J. \& Pizarro, M. A. (1969), Ann. d'Endocrinol. 30,177-182. - 25. KöBBERLING, J. \& v. zUR MüHLEN, A. (1972), diese Z. 10, 67-73. - 26. KöbBERLING, J. \& v. zUR MÜHLEN, A. (in Vorbereitung).
Dr. J. Köbberling 34 Göttingen Humboldtallee 1 